\title{
$\mathrm{BaFe}_{12-x} \mathrm{Ru}_{x} \mathrm{O}_{19}$ 单晶的磁各向异性
}

\author{
刘寄浙 鹿 牧 金通政 王自钧 翟宏如 \\ (南宗大学物理系)
}

若干年来, 为了改进具有实用价值的硬磁材料 $\mathrm{BaFe}_{12} \mathrm{O}_{19}$ (简称 $\mathrm{BaM}$ ) 的磁性, 曾应用离子 置换法进行了许多研究工作, 但是至今尚未在基本磁性上取得明显的进展. 这些研究工作中 的一个重要方面就是用具有强各向异性的过渡族离子来取代 $\mathrm{Fe}^{3+}$ 离子, 从而改变 $\mathrm{BaM}$ 的各向 异性. 已经报道过的这些强各向异性离子有: 第一过渡族中的 $\mathrm{CO}^{2+}{ }^{[1]} 、 \mathrm{Fe}^{2+}{ }^{[2]}$ 及第三过渡族 中的 $\mathrm{Ir}^{4+}{ }^{[3]}$ 等. 实验表明, 所有上述离子在室温下均呈现负的各向异性的贡献, 即 $\Delta K_{1}<0$.

$\mathrm{Ru}^{3+}$ 离子是属于第二过渡族的, 其电子组态为 $4 d^{5}$. 研究表明 ${ }^{[4]}$ 它在尖晶石及石榴石铁氧 体点阵中择优进入八面体晶座, 并表现出强大的各向异性. 但是, 迄今尚末见到有关 $\mathrm{Ru}^{3+}$ 离 子对具有六方结构的 $\mathrm{BaM}$ 的磁性影响的研究报道.

本文研究了少量 $\mathrm{Ru}^{3+}$ 离子添加对 $\mathrm{BaM}$ 单晶磁性的影响. 室温下对 $\mathrm{BaFe}_{12-x} \mathrm{Ru}_{x} \mathrm{O}_{19}$ 单晶 体所进行的磁性测量表明当 $x<0.04$ 时, 单轴各向异性常数 $K_{1}$ 随 $x$ 的增加而急剧增加而 在 $x=0.04$ 时到达极大, 以后则随 $x$ 值的增加而逐渐下降. 其室温下 $K_{1}$ 的最大相对增量为 $\Delta K_{1} / K_{1} \approx 19 \%$.

\section{一、晶体 生长}

所有的晶体均采用助熔剂法生长, 所用的助熔剂为 $\mathrm{Bi}_{2} \mathrm{O}_{3}$. 晶体生长的配方为: 78.7 克 $\mathrm{Bi}_{2} \mathrm{O}_{3}, 7$ 克 $\mathrm{BaCO}_{3}, 16.1$ 克 $\mathrm{Fe}_{2} \mathrm{O}_{3}$ 以及适量的 $\mathrm{Ru}_{2} \mathrm{O}_{3}$. 将上述大约 100 克的原料混合均匀装入 30 - $50 \mathrm{cc}$ 的铂坩埚内, 然后放入顶端加热的碳化矽炉内加热至最高温度 $1300^{\circ} \mathrm{C}$, 在此温度下 保温 20 小时以确保原料熔化均匀. 晶体生长的冷却速率为 $3^{\circ} \mathrm{C} /$ 小时. 当电炉冷却至 $980^{\circ} \mathrm{C}$ 时便切断电源任其自然冷却. 将取出的铂坩埚放入稀硝酸中煮沸, 以便使晶体分离出来. 所 得的晶体用 $\mathrm{X}$ 射线 ( $\mathrm{CoK}$ 辐射) 衍射技术及光谱化学分析进行定性的鉴定. 定量的化学分析 表明, 实际进入样品的 $x$ 值可以偏离原先配方中的加入量 5-10\%.

\section{二、磁 性 测 量}

应用振动样品磁强计对所有成分的晶体进行了室温下磁化强度 $\sigma$ 与磁各向异性“常数 $K_{1}$ 的测定. 所使用的最大场强为 $11 \mathrm{KOe}$. 样品采用直径为 $2 \mathrm{~mm}$ 、厚度约为 $0.1 \mathrm{~mm}$ 的薄圆片. 为 了保证测量的精度, 使用了同样大小的 $\mathrm{Ni}$ 圆片进行定标. 对每一个样品均在平行于 $c$ 轴与垂 直于 $c$ 轴两种情况下测出其 $\sigma_{\|}-H$ 与 $\sigma_{\perp}-H$ 磁化曲线. 室温下磁场为 $11 \mathrm{KOe}$ 下的磁化强度 $\sigma$ 可从 $\sigma_{I I}-H$ 曲线上得出,而各向异性场 $H_{A}=2 K_{1} / \sigma$, 则由 $\sigma_{\perp}-H$ 曲线的直线部分 的 延线与 $\sigma_{\| \prime}-H$ 曲线的交点来确定 ${ }^{[2]}$. 在此, $K_{1}$ 为单轴各向异性常数, 其单位为尔格/克. 由于所测得

本文 1980 年 4 月 24 日收到。

第 1 期

科学通报 
的各向异性场 $H_{A}$ 均大于 $10 \mathrm{KOe}$, 故由于薄圆片的有限厚度所产生的退磁场修正 $(<200 \mathrm{Oe})$ 就可略去不计了.

\section{三、结果与讨论}

图 1 与图 2 为我们在室温下的测量结果. 从图 1 我们可清楚地看出, $\mathrm{BaFe}_{12-x} \mathrm{Ru}_{x} \mathrm{O}_{19}$ (这 里 $x=0,0.01,0.03,0.04,0.05,0.1,0.2,0.35$ 及 0.5 系表示原始配方中的加入量)单晶的 $\sigma$ 值随 $\mathrm{Ru}^{3+}$ 含量的增加而缓慢下降. 图 2 则给出了室温下 $K_{1}$ 值随 $x$ 的变化关系. 由图可以看 出, 当 $x$ 从零逐渐增大时, $K_{1}$ 值随之急剧增加, 在 $x=0.04$ 时到达极大, 其值约为 $7.00 \cdot 10^{5}$ 尔 格/克(对 $\mathrm{BaFe}_{12} \mathrm{O}_{19}, K_{1}=5.85 \cdot 10^{5}$ 尔格/克)， 以后则随 $x$ 的进一步增加而降低. 当 $x=0.5$ 时, 对应的 $K_{1}$ 值降为 $4.86 \cdot 10^{5}$ 尔格/克.

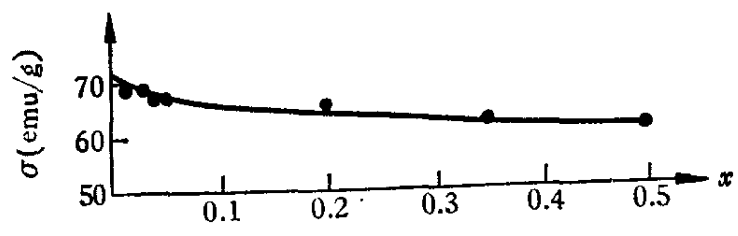

图 $1 \mathrm{BaFe}_{12-x} \mathrm{Ru}_{x} \mathrm{O}_{19}$ 单晶 $\sigma$ 随 $x$ 变化关系 (在 $11 \mathrm{KOe}$ 下测量)

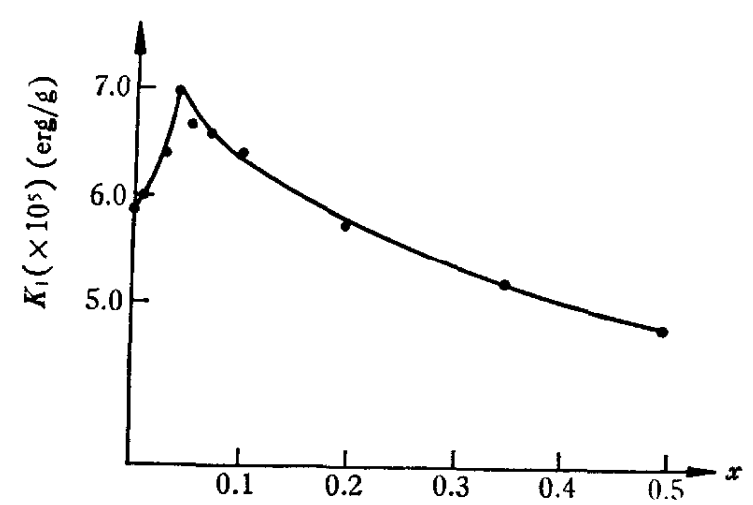

图 $2 \mathrm{BaFe}_{12-x} \mathrm{Ru}_{x} \mathrm{O}_{19}$ 单晶的 $k_{1}-x$ 图

$\mathrm{M}$ 型六角铁氧体 BaM 在结构上属于磁铅石型 (magnetoplumbite) ${ }^{[5]}$. 每个单胞含有两个分 子式. 沿着 $c$ 轴方向,它可看成是由结构形式为 $S R S^{*} R^{*}$ 的 $S$ 块与 $\boldsymbol{R}$ 块的交替系列所组成, 其中 $*$ 表示 $\boldsymbol{S}, \boldsymbol{R}$ 绕 $c$ 轴旋转 $180^{\circ}$. $\boldsymbol{S}$ 块在结构上类同于尖晶石,其 $\langle 111\rangle$ 轴与 $c$ 轴平行. 所 有的铁离子分布在五类不等价的点阵位置上, 其中八面体晶座为 $12 k, 2 a$ 和 $4 f_{2}$, 四面体晶座 是 $4 f_{1}$, 六面体晶座是 $2 b$. 就八面体晶座而言, $2 a$ 位于 $\boldsymbol{S}$ 块中， $4 f_{2}$ 位于 $\boldsymbol{R}$ 块中, 它们的三角 晶场方向均平行于 $c$ 轴. $12 k$ 则位于 $\boldsymbol{R}$ 与 $\boldsymbol{S}$ 块的交界处,其三角晶轴方向偏离 $c$ 轴 $71^{\circ}$. 此 外,从自旋排列的方向来看, $12 k, 2 a$ 与 $2 b$ 座中的自旋方向平行一致,而 $4 f_{1}$ 与 $4 f_{2}$ 座中的自旋 则又彼此平行而指向与前者相反.

按照各向异性的单离子理论，当过渡族离子在尖晶石和石榴石的晶场中的基态呈现简并 态时，由于轨道矩未完全淬灭而可表现出强的各向异性. 以立方晶体中的 $\mathrm{Co}^{2+}$ 为例，在立方 晶场和次近邻三角晶场作用下，其基态沿局部晶场〈111)轴有最大的轨道矩的平均值. 通过自 旋一轨道耦合效应使 $\mathrm{Co}^{2+}$ 表现出以局部〈111〉轴为易磁化方向的强单轴各向异性. Hansen 与 $\mathrm{Krishnan}^{[4]}$ 的研究工作表明, $\mathrm{Ru}^{3+}$ 与 $\mathrm{Ir}^{4+}$ 离子在尖晶石与石榴石铁氧体的八面体中表现出相 类似的特性. $4 d$ 及 $5 d$ 电子在强晶场的作用下表现为低自旋态，且其自旋一轨道耦合系数 $\lambda$ 要比 $3 d$ 电子的为大, 因而具有更强的各向异性, 且亦以局部 $\langle 111\rangle$ 轴为其易磁化方向.

对于六角铁氧体 $\mathrm{BaM}$ 来说, 由于存在着三种类型的八面体座, $12 k, 2 a$ 及 $4 f_{2}$, 因而过渡 族离子的各向异性效应将视其进人的位置的不同而异. Lotgering 等 ${ }^{[1]}$ 在研究 $\mathrm{Co}^{2+} \mathrm{Ti}^{4+}$ 对 $\mathrm{BaM}$ 的磁各向异性的影响时, 认为导致易磁化方向从 $c$ 轴变为 $c$ 面的主要原因是由于 $\mathrm{Co}^{2+}$ 进 入 $12 k$ 位置的结果; 同一作者 ${ }^{[2]}$ 在研究 $\mathrm{LaFe}_{12} \mathrm{O}_{19}$ 的磁性时, 则把 $\mathrm{LaM}$ 与 $\mathrm{BaM}$ 在各向异性上的 不同归因于 $\mathrm{Fe}^{2+}$ 进入了 $2 a$ 座. 在我们的实验中, 当在 $\mathrm{BaM}$ 中掺入微量 $\mathrm{Ru}^{3+}$ 时, 其 $K_{1}$ 值随 $x$ 的增加而上升, 这似乎说明当掺入量很小时, $\mathrm{Ru}^{3+}$ 离子优先进入局部晶轴与 $c$ 轴平行的 $2 a$ 或 
$4 f_{2}$ 位置. 考虑到室温下 $\sigma$ 随 $x$ 增加而减少这一事实, 则我们就可认为 $\mathrm{Ru}^{3+}$ 离子是择优进入 $2 a$ 位置; 而掺入量增加时 $(x>0.04), K_{1}$ 的缓慢下降可能意味着此时 $\mathrm{Ru}^{3+}$ 离子更多地进入了 $12 k$ 位置. 采用这样一个同一各向异性离子可进入不同的八面体晶座从而在对各向异性的贡 献上表现为竞争效应的模型可以很好地从定性上对我们室温下的实验结果加以解释. 为进一 步对上述效应进行深入的研究，我们目前正在对上述成分的样品进行低温下的测量，同时 Mössbauer 效应及定量的成份分析工作也正着手进行.

致谢: 张鸿才同志参加了部份实验工作,特此感谢.

\section{参考文献}

$\lceil 1\rceil$ Lotgering, F. K., Enz, U. \& Smit, J., Philips. Res. Rept., 16(1961), 441.

[2] Lotgering, F. K., J. Phys. Chem. Solids., 16(1974), 1633.

[ 3 T Tauber, A., Kohn, J. A. \& Savage, R. O., J. Appl. Phys., Supplement, 34(1963), 1265.

[ 4 ] Hansen, P. \& Krishnan, R., J. de Physique, supplement, 38(1977), C1-147.

[5] Smit, J. \& Wijn, H. P. J., Ferrites, John Wiley, New York, 1959. 